\title{
Real-Time Nonlinear FEM with Neural Network for Simulating Soft Organ Model Deformation
}

\author{
Ken'ichi Morooka ${ }^{1}$, Xian Chen ${ }^{1}$, Ryo Kurazume ${ }^{2}$, \\ Seiichi Uchida ${ }^{2}$, Kenji Hara ${ }^{3}$, Yumi Iwashita ${ }^{2}$, and Makoto Hashizume ${ }^{1}$ \\ ${ }^{1}$ Digital Medicine Initiative, Kyushu University \\ ${ }^{2}$ Graduate School of Infomation Science and Electrical Engineering, \\ Kyushu University \\ ${ }^{3}$ Faculty of Design, Kyushu University \\ Maidashi 3-1-1, Higashi-ku, Fukuoka 812-8582, Japan \\ morooka@digital.med.kyushu-u.ac.jp
}

\begin{abstract}
This paper presents a new method for simulating the deformation of organ models by using a neural network. The proposed method is based on the idea proposed by Chen et al. 2] that a deformed model can be estimated from the superposition of basic deformation modes. The neural network finds a relationship between external forces and the models deformed by the forces. The experimental results show that the trained network can achieve a real-time simulation while keeping the acceptable accuracy compared with the nonlinear FEM computation.
\end{abstract}

\section{Introduction}

Recent medical imaging devices can provide medical images with high resolutions. Volumetric organ models generated by the images contribute to the improvement of the medical fields. For example, VR-based surgical simulators [1] give surgeons the opportunities of training surgical techniques in various surgery cases. In another case, an endoscopic surgery navigator [6] shows virtual images by fusing volumetric organ models into real endoscopic images. The models contain the internal information of the organs such as the positions of blood vessels and tumors in the organs. Such information can not be obtained from the endoscopic images. We have been studying on the endoscopic surgery navigator.

In actual endoscopic surgery, organs are deformed when surgical instruments touch the organs. According to the deformation of the real organs, the organ models used in the navigator must be deformed in real-time. Moreover, the navigator needs to deal with soft organs which have the properties of anisotropy and inhomogeneity, and undergo large deformation. Many researchers 17] have proposed methods for simulating the soft organ deformation. Among the methods, finite element method (FEM) is a well-known technique for accurately modeling the behaviors of continuous objects. Compared with other simulation techniques, FEM can achieve a more physically realistic simulation for deformable objects. The FEM formulation consists of matrices form derived from the virtual work 


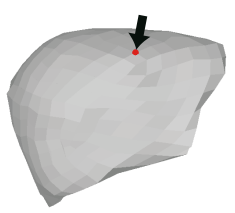

(a)

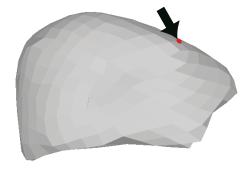

(b)

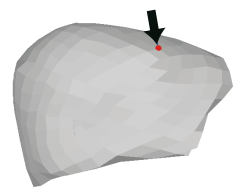

(c)

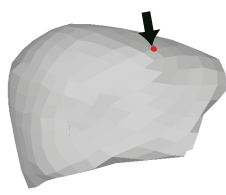

(d)

Fig. 1. New model generated by combination of two deformation modes in 2]. The arrows show the force directions which cause the deformations.

principle or weighted residual method. The terminological classification of linear and nonlinear FEM depends on the treatment of geometrical and material nonlinearities.

The FEM-based simulation is very time-consuming because of solving large scale system of equations. To achieve the real-time simulation, the conventional methods using FEM [35] introduce the assumptions of infinitesimal deformation and linear elasticity. However, the assumptions are applicable to small deformations. In practice, soft organs are deformed greatly in real surgery, and the relationship between such deformations and the resistances to them is nonlinear. If the methods using the linear elastic assumptions are applied with large deformations, the simulation can not provide realistic deformations due to the computational error accumulation. Although there are the simulators based on nonlinear FEM 91011, the computational cost problem still remains in the simulators.

Here, Chen et al. 2] showed the possibility of the nonlinear FEM-based simulation in real-time. They use principle deformed models, called deformation modes. The deformation modes have been computed off-line by considering the nonlinear behavior. Fig[1(a) and (b) show the examples of the deformation modes when an original liver model is pushed in two directions illustrated as the arrows in the figures. In the simulation process, when the resultant of the two forces acts on the model, the deformed model can be predicted by the superposition of the deformation modes. Fig.1(c) shows the model generated by superposing the deformation modes. On the other hand, Fig 1(d) illustrates the model obtained by the nonlinear FEM analysis with the resultant force. Compared with the models, the simulation using the deformation modes allows to reduce the computational cost while keeping the acceptable accuracy of the deformation. However, the authors in [2] don't describe how to realize the method.

This paper proposes a new method for simulating the deformation of a soft organ model based on the superposition of the basic deformation modes. The proposed method employs a neural network to achieve the simulation process. The network learns the relationship between external forces and the model deformations when the forces act on the model. Given an arbitrary force as input data, the trained network, called a neuroFEM, outputs the corresponding deformed model. This paper focuses on the grasping operation of a forceps, which is the instrument used in the endoscopic surgery. 
As well as our method, the methods in 4/812] incorporate neural networks into their deformation simulators. In [48, the networks are used to determine the parameters of mass-spring models. The soft organ deformation in 12 is regarded as the propagation process of a potential energy generated by an external force. A cellular neural network is used to simulate the propagation. However, the method in 12 deals with surface models only.

\section{Notation}

\subsection{Volumetric Organ Model}

We use a volumetric organ model composed of cubic elements and vertices, which are represented in the Cartesian coordinate. When the model contains $N_{v}$ vertices, it is represented by $3 N_{v}$ dimensional vector

$$
\boldsymbol{M}=\left[\boldsymbol{v}_{1}^{T}, \boldsymbol{v}_{2}^{T}, \cdots, \boldsymbol{v}_{N_{v}}^{T}\right]^{T},
$$

where $\boldsymbol{v}_{j}=\left(x_{j}, y_{j}, z_{j}\right)^{T}$ is the $j$-th $\left(j=1, \cdots, N_{v}\right)$ vertex included in the model $\boldsymbol{M}$. A vector (or a matrix) $\boldsymbol{A}^{T}$ means the transpose of $\boldsymbol{A}$.

Given two models $\boldsymbol{M}$ and $\boldsymbol{M}^{\prime}$, the difference of $\boldsymbol{M}^{\prime}$ to $\boldsymbol{M}$ is defined by the Euclidean distance between the models:

$$
D\left(\boldsymbol{M}^{\prime} \mid \boldsymbol{M}\right)=\frac{1}{\mu}\left\|\boldsymbol{M}-\boldsymbol{M}^{\prime}\right\|,
$$

where $\|\cdot\|$ denotes the Euclidean distance. The parameter $\mu$ is the representative size of the model $\boldsymbol{M}$, and is set to the diagonal line length of the bounding box of $\boldsymbol{M}$. Since an arbitrary organ morphology varies in individual, it is difficult to determine the general size and shape of the organ. Therefore, we simply represent the model size by the bounding box of the model.

\subsection{Nonlinear FEM Analysis by Using Deformation Modes}

This section verifies the validity of the nonlinear FEM computation by using the deformation modes. First, we randomly select a pair of two initial external forces, $\boldsymbol{f}_{0}$ and $\boldsymbol{f}_{1}$. The resultant force $\boldsymbol{f}_{2}$ is defined as $\boldsymbol{f}_{2}=\left(\boldsymbol{f}_{0}+\boldsymbol{f}_{1}\right) / 2$. The nonlinear FEM simulates the model deformation caused by $\boldsymbol{f}_{0}, \boldsymbol{f}_{1}$ and $\boldsymbol{f}_{2}$, and outputs the models $\boldsymbol{M}_{0}, \boldsymbol{M}_{1}$, and $\boldsymbol{M}_{2}$, respectively. The another model $\boldsymbol{M}^{\prime}$ is generated by the weighted linear combination of $\boldsymbol{M}_{0}$ and $\boldsymbol{M}_{1}$ :

$$
\boldsymbol{M}^{\prime}=\alpha \boldsymbol{M}_{0}+(1-\alpha) \boldsymbol{M}_{1} .
$$

The weight parameter $\alpha(0 \leq \alpha \leq 1)$ in eq (3) is determined by

$$
\alpha=\arg \min _{\alpha} D\left(\boldsymbol{M}^{\prime} \mid \boldsymbol{M}_{2}\right) .
$$

Finally, we calculate the difference of $\boldsymbol{M}^{\prime}$ to $\boldsymbol{M}_{2}$ by eq. (2). In practice, we choose 500 pairs of the initial external forces, and the average difference is $1.41 \times 10^{-5}$. The result shows that the superposition of deformation modes is useful for the real-time nonlinear FEM simulation. 


\section{Methods}

\subsection{Training Data Set}

Generally, a neural network trains by observing many training data. In our method, each training data is a pair of an external force and its corresponding deformation mode, which is the model deformed by the force. An external force is regarded as a set of three elements; the contact point, the unit directional vector and the magnitude of the force. All the vertices on the model surface are used as the contact point of the force. Using a vector $\boldsymbol{y}$ generated by a random number generator. the magnitude and the directional vector of the force are calculated by the norm $\|\boldsymbol{y}\|$ and the unit vector $\frac{\boldsymbol{y}}{\|\boldsymbol{y}\|}$ of $\boldsymbol{y}$, respectively.

Many deformation modes are generated by changing the force elements. Each neuron in the output layer corresponds to each element of $3 N_{v}$ dimensional model $\boldsymbol{M}$, meaning that the output layer has $3 N_{v}$ neurons. For example, in the case of our liver model with $1 \mathrm{~K}$ vertices, the output layer needs $3 \mathrm{~K}$ neurons while the input layer contains 7 neurons. Since the training of such network needs many neurons, the direct use of the model leads to much computational cost. To speed up the training process, we compress the data size of the modes by principle components analysis (PCA).

Using all the deformation modes, the matrix $\boldsymbol{X}$ is obtained by

$$
\begin{aligned}
& \boldsymbol{X}=\boldsymbol{Y}^{T} \boldsymbol{Y} ; \quad \boldsymbol{Y}=\left[\left(\boldsymbol{M}_{1}-\overline{\boldsymbol{M}}\right),\left(\boldsymbol{M}_{2}-\overline{\boldsymbol{M}}\right), \cdots\left(\boldsymbol{M}_{N}-\overline{\boldsymbol{M}}\right)\right] \\
& \overline{\boldsymbol{M}}=\frac{1}{N_{M}} \sum_{i=1}^{N_{M}} \boldsymbol{M}_{i}
\end{aligned}
$$

where $\overline{\boldsymbol{M}}$ is called the average deformation mode, and $\boldsymbol{M}_{i}$ is the $i$-th $(i=$ $\left.1, \cdots, N_{M}\right)$ deformation mode. The eigen vectors of $\boldsymbol{X}$ are calculated by applying PCA to $\boldsymbol{X}$. Each eigen vector has its eigen value which means the distribution of the deformation modes along the eigen vector. We sort all the eigen vectors in descending order of their eigen values. The first $s\left(s<N_{v}\right)$ eigen vectors are selected so that their cumulative contribution ratio $R(s)$ is satisfied with

$$
R(s)=\frac{\sum_{l=1}^{s} e_{l}}{\sum_{l^{\prime}=1}^{3 N_{v}} e_{l^{\prime}}} \geq \tau,
$$

where $e_{l}$ is the eigen value of $l$-th eigen vector. The value of a threshold $\tau$ is set to $\tau=0.9999$ in our experiments. The selected eigen vectors form the projection matrix $\boldsymbol{\Phi}$ defined as $\boldsymbol{\Phi}=\left[\begin{array}{llll}\boldsymbol{u}_{1} & \boldsymbol{u}_{2} & \cdots & \boldsymbol{u}_{s}\end{array}\right]^{T}$. The matrix $\boldsymbol{\Phi}$ can convert the $3 N_{v^{-}}$ dimensional deformation mode $\boldsymbol{M}$ into a lower $s$-dimensional vector $\boldsymbol{M}^{*}$ :

$$
M^{*}=\boldsymbol{\Phi}(\boldsymbol{M}-\bar{M}) .
$$

All the deformation modes are converted into $s$-dimensional vectors by eq.(17). 


\subsection{NeuroFEM Construction}

Given input and output data, a neural network trains to model the relationship between the input and output data. The trained network, called a neuroFEM, automatically generalizes. In other words, even though input data that are not included in the training data are given, the network can estimate the appropriate output by interpolating between the training data. This character is suitable for our neuroFEM because the proposed method is also based on the combination of the deformation modes.

We use a multilayer neural network composed of multiple layers. The first and last layers are referred to as the input and the output layers, respectively. The intermediate layers are hidden layers. Each layer has the set of neurons, and a neuron is connected to all the neurons in the subsequent layer. When there are $L_{k}$ neurons included in the $k$-th $\left(k=1, \cdots, N_{k}\right)$ layer and the $n$-th neuron in the $k$-th layer receives a signal $x_{n}^{k}$, the neuron outputs a signal $o_{n}^{k}$

$$
o_{n}^{k}=\frac{1}{1+\exp \left(-\epsilon x_{n}^{k}\right)} ; \quad x_{n}^{k}=\sum_{m=1}^{L_{k-1}+1} w_{m, n}^{k-1, k} o_{m}^{k-1},
$$

where $w_{m, n}^{k-1, k}$ is the connection weight between the $m$-th neuron in the $(k-1)$-th layer and the $n$-th neuron in the $k$-th layer. We set the parameter $\epsilon$ in eq.(8) to $\epsilon=1.0$ in our experiments.

In our method, the error $E$ of the network is defined by

$$
E=\frac{1}{N_{M}} \sum_{i=1}^{N_{M}} \sum_{n=1}^{s}\left(c_{n}^{i}-o_{n}^{N_{k}}(i)\right)^{2},
$$

where $c_{n}^{i}$ is the $n$-th element of the deformation mode in the $i$-th training data. The signal $o_{n}^{N_{k}}(i)$ is the output of the $n$-th neuron in the output layer when the $i$-th training data is given. Using the back-propagation algorithm, the network trains by adjusting all the connection weights in the network. The training process is repeated until the error is less than a given threshold.

Generally, a neural network can solve the complex problem when the network consists of many hidden layers and/or many neurons in the hidden layer. Moreover, the neuroFEM can treat models with many vertices by applying PCA to the model to compress the model data size. Therefore, there is no limitation concerning the model used in our method. On the contrary, there is no way for finding the suitable number of the hidden layers and their neurons to solve an arbitrary problem. We empirically determined the number of the hidden layers and elements in each layer.

\subsection{NeuroFEM-Based Simulator}

During the grasping simulation, through a mouse or a haptic device, users control a virtual laparoscopic forceps and grasp the liver model by the forceps. Let us consider that the forceps grasps the surface of the organ model. First, the vertex 


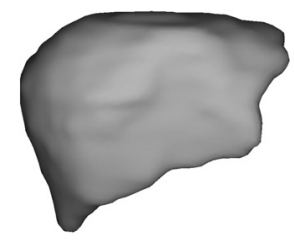

Fig. 2. Original liver model

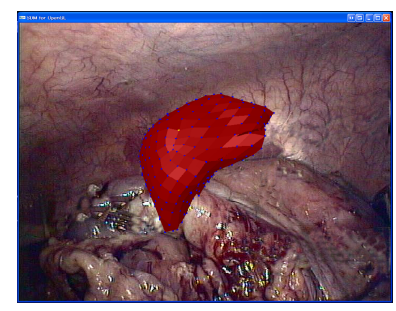

Fig. 3. Screenshot of the neuroFEM-based simulator

closest to the grasp position is selected from all the vertices on the model surface. The selected vertex is called a reference vector $\boldsymbol{r}$.

When the forceps keeps grasping the reference vertex and moves toward an arbitrary location $\boldsymbol{q}^{(t)}$ at a time $t$, a current external force $\boldsymbol{f}^{(t)}$ is determined as follows. We assume that the material property of the organ is elasticity except viscosity and plasticity. On the assumption, the position of the reference vector is used as the contact position $\boldsymbol{p}^{(t)}$ of the force $\boldsymbol{f}^{(t)}$. The direction $\boldsymbol{d}^{(t)}$ and the magnitude $h^{(t)}$ of $\boldsymbol{f}^{(t)}$ is calculated by the following equations:

$$
\boldsymbol{d}^{(t)}=\frac{\boldsymbol{q}^{(t)}-\boldsymbol{r}}{\left\|\boldsymbol{q}^{(t)}-\boldsymbol{r}\right\|} ; \quad h^{(t)}=\left\|\boldsymbol{q}^{(t)}-\boldsymbol{r}\right\| .
$$

Using the force $\boldsymbol{f}^{(t)}=\left[\boldsymbol{p}^{(t)}, \boldsymbol{d}^{(t)}, h^{(t)}\right]$ as the input, the neuroFEM outputs the $s$ dimensional vector $\boldsymbol{M}^{*}$. From eq.(7), we obtain the resulting model $\boldsymbol{M}$ by back-projecting the vector $\boldsymbol{M}^{*}$ into the original model space;

$$
M=\Phi^{-1} M^{*}+\bar{M}
$$

where $\boldsymbol{\Phi}^{-1}$ is the inverse matrix of the projection matrix $\boldsymbol{\Phi}$, and can be replaced by the transpose $\boldsymbol{\Phi}^{T}$ of $\boldsymbol{\Phi}$.

If the grasp position is changed, we need to update the reference vector. The vertex $\boldsymbol{v}^{*}$ closest to the latest grasp position is selected from all the vertices on the model surface. We find the position $\tilde{\boldsymbol{v}}^{*}$ of the vertex $\boldsymbol{v}^{*}$ in the model with its original shape. The reference vector is replaced by $\tilde{\boldsymbol{v}}^{*}$.

\section{Experimental Results}

To verify the applicability of the proposed method, we conducted the experiment using the volume model of a liver as shown in Fig. 2. The liver model consists of 729 vertices and 512 cubic elements. The neuroFEM is implemented on a PC platform (Pentium4 2.8 GHz with $1 \mathrm{G} \mathrm{MB}$ ).

In the experiments, 10,314 deformation modes are generated. The bottom row in Fig. 4 (a) and (b) show the examples of the deformation modes caused by two types of the forces, $\boldsymbol{f}_{1}$ and $\boldsymbol{f}_{2}$, respectively. All deformation modes are converted 

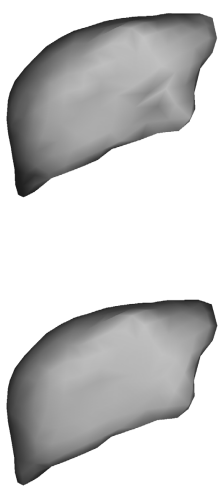

(a)
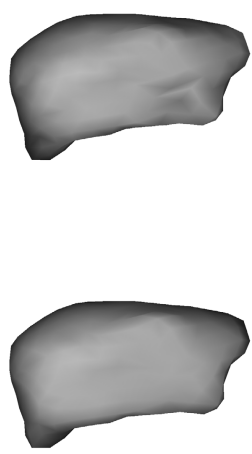

(b)
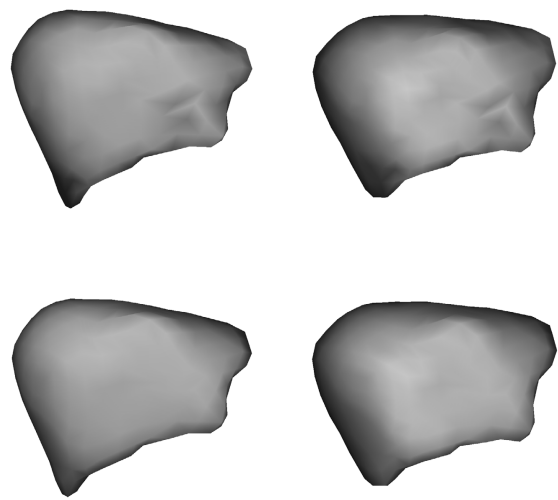

(c)

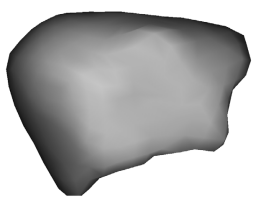

(d)

Fig. 4. Comparison of our method (top row) with the nonlinear FEM (bottom row). The models in (a) and (b) are generated by two forces included in the training data set while the models in (c) and (d) are generated when arbitrary forces act on the original model.

into 13 dimension vectors by PCA described in section 3.1. The network used in our method consists of an input layer, 2 hidden layers and an output layer. Since the force parameters are used as the input data of the network, there are 7 units in the input layer. The output of the network are the corresponding position of a deformed model in the eigenspace. Therefore, the output layer has 13 neurons. The final error in eq.(9) is $9.5 \times 10^{-5}$. when the training process is terminated. The top row in Fig. 4 (a) and (b) show the deformed models obtained by the network. Then, the same forces $\boldsymbol{f}_{1}$ and $\boldsymbol{f}_{2}$ are used as the input of the network.

We compare the proposed method with the original nonlinear FEM analysis. The comparison uses as the input data 380 new forces which are not included in the training data. Fig. 4 (c) and (d) show the examples of the deformed models obtained by our method (top row) and nonlinear FEM (bottom row), respectively. In the neuroFEM, it takes 0.28 [msec] to output the final model after an input data is given. The computational time of the neuroFEM is about 1,000 times faster than that of the nonlinear FEM. Also we calculated the differences between the models obtained by the neuroFEM and the nonlinear FEM. The difference between two models is defined by eq.(2), and the average difference is 0.09. From the results, the performance of our method is almost equal that of nonlinear FEM, and our method can reduce the computational time.

\section{Conclusion}

This paper presented a method for simulating the deformation of organ models by the neural network, called the neuroFEM. The network learned a relationship between forces and the deformation modes when the forces act on the organ model. As a result, the neuroFEM can emulate the deformation simulator using 
nonlinear FEM. Some experimental results show that our method can achieve the real-time nonlinear FEM simulation of deforming the model with acceptable accuracy compared with nonlinear FEM. Generally, the FEM computation cost exponentially increases according to the number of the vertices included in the model. On the contrary, the neuroFEM outputs the model by computing the simple and linear equations (8) and (11). Since these equations are independent of the data size of the model, our method is a powerful tool when models with a large number of vertices are used in the simulation. Now we have been developing the extension of the neuroFEM for topological changing of the model.

\section{References}

1. Basdogan, C., Sedef, M., Harders, M., Wesarg, S.: VR-based simulators for training in minimally invasive surgery. IEEE Comput. Graph. Appl. 27, 54-56 (2007)

2. Chen, X., Hisada, T., Sakuma, I., Dohi, T., Shimada, M., Hashizume, M.: Study on liver surgery navigation based on nonlinear finite element method. J. Japan Society of Computer Aided Surgery 5, 15-22 (2003) (In Japanese)

3. Cotin, S., Delingette, H., Ayache, N.: A hybrid elastic model for real-time cutting, deformations, and force feedback for surgery training and simulation. The Visual Computer 16, 437-452 (2000)

4. Duysak, A., Zhang, J., Ilankovan, V.: Efficient modelling and simulation of soft tissue deformation using mass-spring systems. International Congress Series 1256, 337-342 (2003)

5. Inoue, Y., Masutani, Y., Ishii, K., Kumai, N., Kimura, F., Sakuma, I.: Development of surgical simulator with high-quality visualization based on finite-element method and deformable volume rendering. Systems and Computers in Japan 37, 67-76 (2006)

6. Konishi, K., Nakamoto, M., Kakeji, Y., Tanoue, K., Kawanaka, H., Yamaguchi, S., Ieiri, S., Sato, Y., Maehara, Y., Tamura, S., Hashizume, M.: A real-time navigation system for laparoscopic surgery based on three-dimensional ultrasound using magneto-optic hybrid tracking configuration. Int. J. of Computer Assisted Radiology and Surgery 2, 1-10 (2007)

7. Meier, U., López, O., Monserrat, C., Juan, M.C., Alcañiz, M.: Real-time deformable models for surgery simulation: a survey. Computer Methods and Programs in Biomedicine 77, 183-197 (2005)

8. Nürnberger, A., Radetzky, A., Kruse, R.: Using recurrent neuro-fuzzy techniques for the identification and simulation of dynamic systems. Neurocomputing 36, 1-4 (2001)

9. Picinbono, G., Delingette, H., Ayache, N.: Non-linear anisotropic elasticity for realtime surgery simulation. Graphical Models 65, 305-321 (2003)

10. Szèakely, G., Brechbüehler, C., Hutter, R., Rhomberg, A., Schmid, P.: Modeling of soft tissue deformation for laparoscopic surgery simulation. Medical Image Analysis $4,57-66(2000)$

11. Wu, X., Downes, M.S., Goktekin, T., Tendick, F.: Adaptive nonlinear finite elements for deformable body simulation using dynamic progressive mesh. Computer Graphics Forum 20, 349-358 (2001)

12. Zhong, Y., Shirinzadeh, B., Alici, G., Smith, J.: A cellular neural network methodology for deformable object simulation. IEEE Trans. Inf. Technol. Biomed. 10, 749-762 (2006) 